\title{
Novel Genetic Characterization of Porcine Epidemic Diarrhoea Virus Strains Circulating in Guangdong, China
}

\author{
Shengnan Chen ${ }^{1}$, Guangzhi Yan ${ }^{1}$, Tian Tang ${ }^{1}$, Rusen Deng ${ }^{1}$, Xiao qing Yuan ${ }^{1}$, Jiedan \\ Liao $^{1}$, Xing-kui $\mathrm{Si}^{1}$, Zongji Lu ${ }^{1}$, Ningyi $\mathrm{Jin}^{2}$, Hao Liu ${ }^{1}$, and Liangzong Huang ${ }^{1}$ \\ ${ }^{1}$ Foshan University \\ ${ }^{2}$ Academy of Military Sciences
}

October 12,2020

\begin{abstract}
In recent years, the emergence and high prevalence of porcine epidemic diarrhoea virus (PEDV) in piglets has been observed in various regions of Guangdong Province. In this study, novel genetic features of the PEDV S1 gene were detected in fifty-five PEDV samples from eleven different pig farms collected from 2018 to 2019 in eight cities in Guangdong Province, China. More than $98.2 \%(54 / 55)$ of the samples tested positive with a PEDV antigen assay. The S1 gene of 11 samples was sequenced. Analysis results showed that the four novel PEDV isolates were $99.9 \% \sim 100 \%$ identical to each other and clustered to a separate clade in the G2a subtype, sharing $90.4-98.8 \%$ and $87.7-97.7 \%$ identities at the nucleotide and amino acid levels, respectively, with 57 strains from GenBank. It is worth noting that the novel PEDV strains contained nine novel amino acid substitutions (L299I, V312A, Q319P, N/D360A, S558L, S566K, K593R, Y612H and S773F) in the S1 protein compared to the sequences of widely used vaccine strains (CV777 and AJ1102 strains) and other variant PEDV strains (G2 group strains). Furthermore, the amino acid substitutions were in the COE region of the important S protein neutralization epitope. In this study, we detected novel PEDV epidemic strains in Guangdong Province, which had the highest identity (98.7-98.8\% nucleotide level, 96.6-97.2\% amino acid level) with the BJ2011-1 strain and differed greatly from vaccine strains. Compared to vaccine strains, there are 91 (CV777) or 27 (AJ1102) amino acid changes in the neutralization epitope of the S1 protein, and whether amino acid substitutions affect the immune efficacy of the vaccine should be verified in further studies.
\end{abstract}

\section{Hosted file}

Novel Genetic Characterization of Porcine Epidemic Diarrhoea Virus Strains Circulating in Guangdong, Ch available at https://authorea.com/users/366541/articles/486268-novel-geneticcharacterization-of-porcine-epidemic-diarrhoea-virus-strains-circulating-in-guangdongchina

\section{Hosted file}

Table 1.pdf available at https://authorea.com/users/366541/articles/486268-novel-geneticcharacterization-of-porcine-epidemic-diarrhoea-virus-strains-circulating-in-guangdongchina 


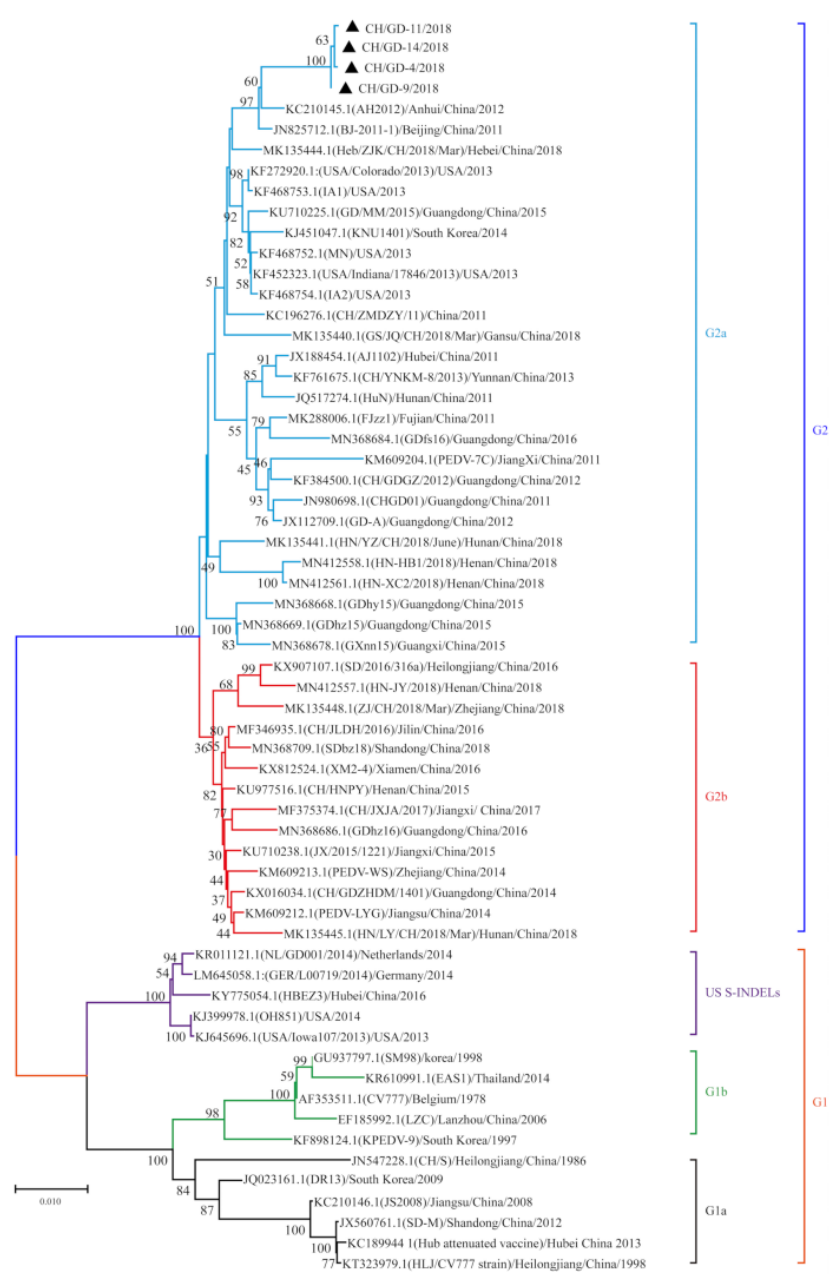




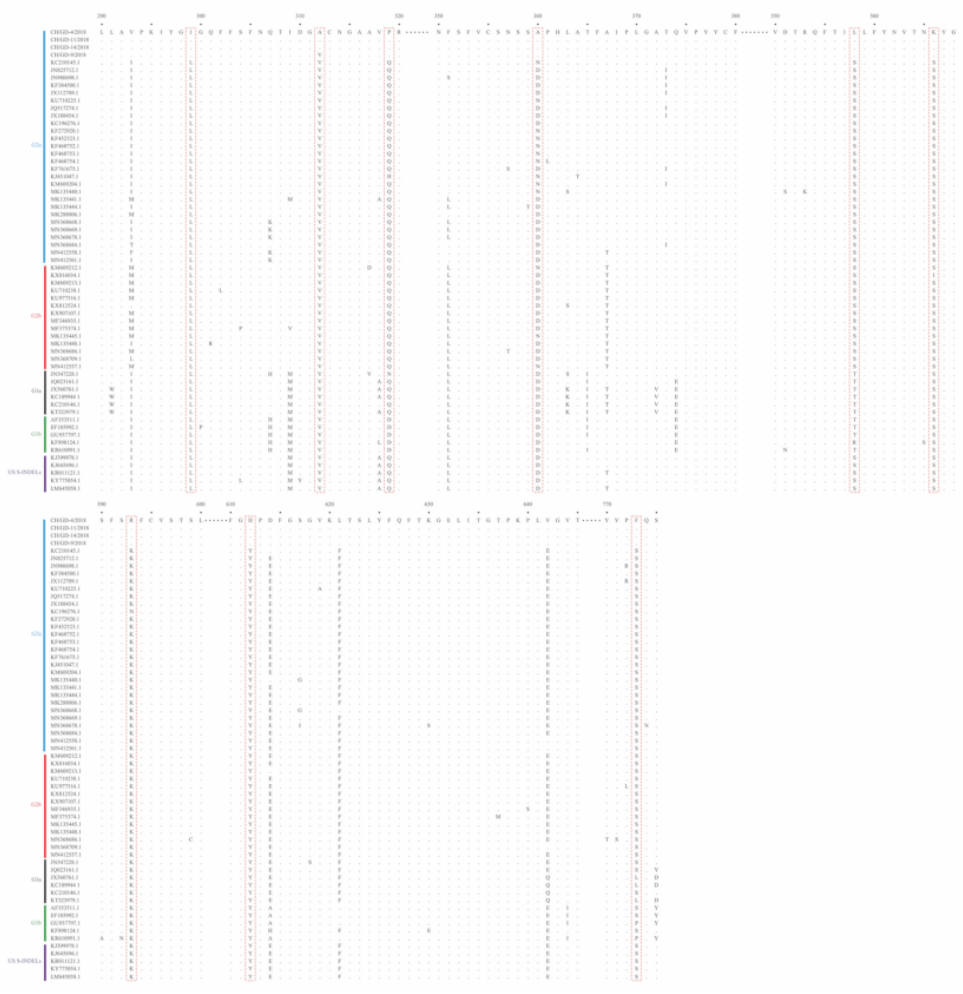




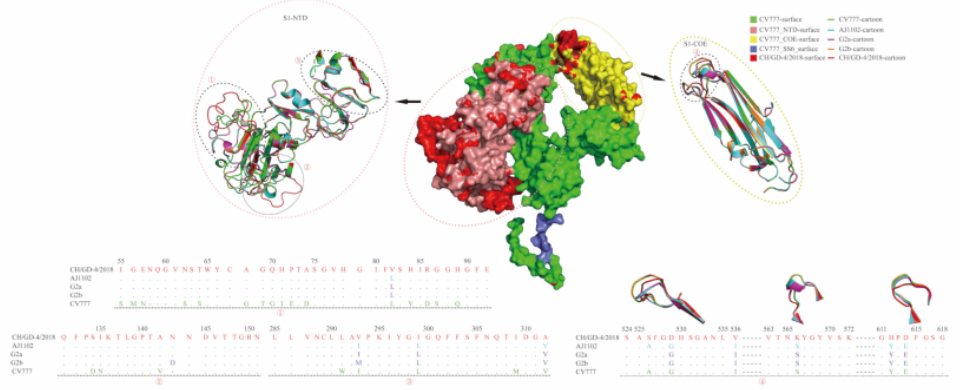

